Bull. Korean Math. Soc. 52 (2015), No. 3, pp. 1007-1025

http://dx.doi.org/10.4134/BKMS.2015.52.3.1007

\title{
AN ANALOGUE OF THE HILTON-MILNER THEOREM FOR WEAK COMPOSITIONS
}

\author{
Cheng Yeaw Ku and KoK Bin Wong
}

\begin{abstract}
Let $\mathbb{N}_{0}$ be the set of non-negative integers, and let $P(n, l)$ denote the set of all weak compositions of $n$ with $l$ parts, i.e., $P(n, l)=$ $\left\{\left(x_{1}, x_{2}, \ldots, x_{l}\right) \in \mathbb{N}_{0}^{l}: x_{1}+x_{2}+\cdots+x_{l}=n\right\}$. For any element $\mathbf{u}=$ $\left(u_{1}, u_{2}, \ldots, u_{l}\right) \in P(n, l)$, denote its $i$ th-coordinate by $\mathbf{u}(i)$, i.e., $\mathbf{u}(i)=u_{i}$. A family $\mathcal{A} \subseteq P(n, l)$ is said to be $t$-intersecting if $|\{i: \mathbf{u}(i)=\mathbf{v}(i)\}| \geq t$ for all $\mathbf{u}, \mathbf{v} \in \mathcal{A}$. A family $\mathcal{A} \subseteq P(n, l)$ is said to be trivially $t$-intersecting if there is a $t$-set $T$ of $[l]=\{1,2, \ldots, l\}$ and elements $y_{s} \in \mathbb{N}_{0}(s \in T)$ such that $\mathcal{A}=\left\{\mathbf{u} \in P(n, l): \mathbf{u}(j)=y_{j}\right.$ for all $\left.j \in T\right\}$. We prove that given any positive integers $l, t$ with $l \geq 2 t+3$, there exists a constant $n_{0}(l, t)$ depending only on $l$ and $t$, such that for all $n \geq n_{0}(l, t)$, if $\mathcal{A} \subseteq P(n, l)$ is
\end{abstract} non-trivially $t$-intersecting, then

$$
|\mathcal{A}| \leq\left(\begin{array}{c}
n+l-t-1 \\
l-t-1
\end{array}\right)-\left(\begin{array}{c}
n-1 \\
l-t-1
\end{array}\right)+t .
$$

Moreover, equality holds if and only if there is a $t$-set $T$ of $[l]$ such that

$$
\mathcal{A}=\bigcup_{s \in[l] \backslash T} \mathcal{A}_{s} \cup\left\{\mathbf{q}_{i}: i \in T\right\},
$$

where

$$
\mathcal{A}_{s}=\{\mathbf{u} \in P(n, l): \mathbf{u}(j)=0 \text { for all } j \in T \text { and } \mathbf{u}(s)=0\}
$$

and $\mathbf{q}_{i} \in P(n, l)$ with $\mathbf{q}_{i}(j)=0$ for all $j \in[l] \backslash\{i\}$ and $\mathbf{q}_{i}(i)=n$.

\section{Introduction}

Let $[n]=\{1, \ldots, n\}$, and let $\left(\begin{array}{c}{[n]} \\ k\end{array}\right)$ denote the family of all $k$-subsets of $[n]$. A family $\mathcal{A}$ of subsets of $[n]$ is $t$-intersecting if $|A \cap B| \geq t$ for all $A, B \in \mathcal{A}$. One of the most beautiful results in extremal combinatorics is the Erdős-Ko-Rado theorem.

Theorem 1.1 (Erdős, Ko, and Rado [14], Frankl [15], Wilson [43]). Suppose $\mathcal{A} \subseteq\left(\begin{array}{c}{[n]} \\ k\end{array}\right)$ is t-intersecting and $n>2 k-t$. Then for $n \geq(k-t+1)(t+1)$, we

Received July 1, 2014; Revised September 30, 2014.

2010 Mathematics Subject Classification. 05D05.

Key words and phrases. cross-intersecting family, Hilton-Milner, Erdős-Ko-Rado, weak compositions. 
have

$$
|\mathcal{A}| \leq\left(\begin{array}{l}
n-t \\
k-t
\end{array}\right)
$$

Moreover, if $n>(k-t+1)(t+1)$, then equality holds if and only if $\mathcal{A}=\{A \in$ $\left.\left(\begin{array}{c}{[n]} \\ k\end{array}\right): T \subseteq A\right\}$ for some t-set $T$.

In the celebrated paper [1], Ahlswede and Khachatrian extended the ErdösKo-Rado theorem by determining the structure of all $t$-intersecting set systems of maximum size for all possible $n$ (see also $[3,17,25,29,37,39,40,41]$ for some related results). There have been many recent results showing that a version of the Erdös-Ko-Rado theorem holds for combinatorial objects other than set systems. For example, an analogue of the Erdős-Ko-Rado theorem for the Hamming scheme is proved in [38]. A complete solution for the $t$ intersection problem in the Hamming space is given in [2]. Intersecting families of permutations were initiated by Deza and Frankl in [10]. Some recent work done on this problem and its variants can be found in $[5,7,8,11,12,19,26$, $33,34,35,36,42]$. The investigation of the Erdős-Ko-Rado property for graphs started in [23], and gave rise to [4, 6, 21, 22, 24, 44]. The Erdős-Ko-Rado type results also appear in vector spaces [9, 18], set partitions [27, 28, 31] and weak compositions [30, 32].

For a family $\mathcal{A}$ of $k$-subsets, $\mathcal{A}$ is said to be trivially $t$-intersecting if there exists a $t$-set $T=\left\{x_{1}, \ldots, x_{t}\right\}$ such that all members of $\mathcal{A}$ contain $T$. The Erdös-Ko-Rado theorem implies that a $t$-intersecting family of maximum size must be trivially $t$-intersecting when $n$ is sufficiently large in terms of $k$ and $t$.

Hilton and Milner [20] proved a strengthening of the Erdős-Ko-Rado theorem for $t=1$ by determining the maximum size of a non-trivial 1-intersecting family. A short and elegant proof was later given by Frankl and Füredi [16] using the shifting technique.

Theorem 1.2 (Hilton-Milner). Let $\mathcal{A} \subseteq\left(\begin{array}{c}{[n]} \\ k\end{array}\right)$ be a non-trivial 1-intersecting family with $k \geq 4$ and $n>2 k$. Then

$$
|\mathcal{A}| \leq\left(\begin{array}{c}
n-1 \\
k-1
\end{array}\right)-\left(\begin{array}{c}
n-k-1 \\
k-1
\end{array}\right)+1
$$

Equality holds if and only if

$$
\mathcal{A}=\left\{X \in\left(\begin{array}{c}
{[n]} \\
k
\end{array}\right): x \in X, X \cap Y \neq \emptyset\right\} \cup\{Y\}
$$

for some $k$-subset $Y \in\left(\begin{array}{c}{[n]} \\ k\end{array}\right)$ and $x \in X \backslash Y$.

In this paper, we prove an analogue of Theorem 1.2 for weak compositions with fixed number of parts. Let $\mathbb{N}_{0}$ be the set of non-negative integers, and let $P(n, l)$ denote the set of all weak compositions of $n$ with $l$ parts, i.e., $P(n, l)=$ $\left\{\left(x_{1}, x_{2}, \ldots, x_{l}\right) \in \mathbb{N}_{0}^{l}: x_{1}+x_{2}+\cdots+x_{l}=n\right\}$. Recall that

$$
|P(n, l)|=\left(\begin{array}{c}
n+l-1 \\
l-1
\end{array}\right) .
$$


For any element $\mathbf{u}=\left(u_{1}, u_{2}, \ldots, u_{l}\right) \in P(n, l)$, denote its $i$ th-coordinate by $\mathbf{u}(i)$, i.e., $\mathbf{u}(i)=u_{i}$. A family $\mathcal{A} \subseteq P(n, l)$ is said to be t-intersecting if $|\{i: \mathbf{u}(i)=\mathbf{v}(i)\}| \geq t$ for all $\mathbf{u}, \mathbf{v} \in \mathcal{A}$.

A family $\mathcal{A} \subseteq P(n, l)$ is said to be trivially $t$-intersecting if there is a $t$-set $T$ of $[l]$ and elements $y_{s} \in \mathbb{N}_{0}(s \in T)$ such that $\mathcal{A}=\{\mathbf{u} \in P(n, l): \mathbf{u}(j)=$ $y_{j}$ for all $\left.j \in T\right\}$. If $y_{j}=0$ for all $j \in T$, then $\mathcal{A}$ is said to be strong-trivially $t$-intersecting. It has been shown that a $t$-intersecting family of maximum size must be strong-trivially $t$-intersecting when $n$ is sufficiently large in terms of $l$ and $t$ [30, Theorem 1.2]. Similar problems have been considered by Engel and Frankl in [13] for $t$-intersecting families in $E_{l}(k, n)$, where the roles of $l$ and $n$ are interchanged from the notation in this paper. Although the case $k=l$ corresponds to $P(n, l)$, they did not address this case in their paper.

Our main result is the following.

Theorem 1.3. Given any positive integers $l, t$ with $l \geq 2 t+3$, there exists a constant $n_{0}(l, t)$ depending only on $l$ and $t$, such that for all $n \geq n_{0}(l, t)$, if $\mathcal{A} \subseteq P(n, l)$ is non-trivially t-intersecting, then

$$
|\mathcal{A}| \leq\left(\begin{array}{c}
n+l-t-1 \\
l-t-1
\end{array}\right)-\left(\begin{array}{c}
n-1 \\
l-t-1
\end{array}\right)+t
$$

Moreover, equality holds if and only if there is a t-set $T$ of $[l]$ such that

$$
\mathcal{A}=\bigcup_{s \in[l] \backslash T} \mathcal{A}_{s} \cup\left\{\mathbf{q}_{i}: i \in T\right\}
$$

where

$$
\mathcal{A}_{s}=\{\mathbf{u} \in P(n, l): \mathbf{u}(j)=0 \text { for all } j \in T \text { and } \mathbf{u}(s)=0\}
$$

and $\mathbf{q}_{i} \in P(n, l)$ with $\mathbf{q}_{i}(j)=0$ for all $j \in[l] \backslash\{i\}$ and $\mathbf{q}_{i}(i)=n$.

Note that

$$
\bigcup_{s \in[l] \backslash T} \mathcal{A}_{s}=\{\mathbf{u} \in P(n, l): \mathbf{u}(j)=0 \text { for all } j \in T\} \backslash\{\mathbf{u} \in P(n, l): \mathbf{u}(j)=0
$$

for all $j \in T$ and $\mathbf{u}(s) \neq 0$ for all $s \in[l] \backslash T\}$.

Therefore,

$$
\left|\bigcup_{s \in[l] \backslash T} \mathcal{A}_{s}\right|=\left(\begin{array}{c}
n+l-t-1 \\
l-t-1
\end{array}\right)-\left(\begin{array}{c}
n-1 \\
l-t-1
\end{array}\right) .
$$

\section{Certain intersecting conditions}

The aim of this section is to prove Corollary 2.6 which will be used later in the proof of the main result. We first need some technical lemmas.

Lemma 2.1. Let $m$ be a positive integer, $r \geq 3$, and $k \in[r-2]$. Let $y_{1}, y_{2}, \ldots, y_{k} \in \mathbb{N}_{0}$ and

$$
\mathcal{C}=\left\{\mathbf{u} \in P(m, r): \mathbf{u}(i)=y_{i} \text { for all } i \in[k-1] \text { and } \mathbf{u}(k)=0\right\},
$$




$$
\mathcal{D}=\left\{\mathbf{u} \in P(m, r): \mathbf{u}(i)=y_{i} \text { for all } i \in[k-1] \text { and } \mathbf{u}(k)=y_{k}\right\} .
$$

Then

(a) $\mathcal{D}=\mathcal{C}$ if $m-\sum_{i=1}^{k-1} y_{i}<0$ or $y_{k}=0$;

(b) $|\mathcal{D}|<|\mathcal{C}|$ if $m-\sum_{i=1}^{k-1} y_{i} \geq 0$ and $y_{k}>0$.

Proof. If $y_{k}=0$, then $\mathcal{D}=\mathcal{C}$. If $m-\sum_{i=1}^{k-1} y_{i}<0$, then $\mathcal{D}=\mathcal{C}=\varnothing$. Suppose $m-\sum_{i=1}^{k-1} y_{i} \geq 0$ and $y_{k}>0$. Note that

$$
|\mathcal{C}|=\left(\begin{array}{c}
m-\sum_{i=1}^{k-1} y_{i}+r-k-1 \\
r-k-1
\end{array}\right) .
$$

If $m-\sum_{i=1}^{k} y_{i}<0$, then $\mathcal{D}=\varnothing$. So, $|\mathcal{D}|<|\mathcal{C}|$. If $m-\sum_{i=1}^{k} y_{i} \geq 0$, then

$$
\begin{aligned}
|\mathcal{D}| & =\left(\begin{array}{c}
m-\sum_{i=1}^{k} y_{i}+r-k-1 \\
r-k-1
\end{array}\right) \\
& <\left(\begin{array}{c}
m-\sum_{i=1}^{k-1} y_{i}+r-k-1 \\
r-k-1
\end{array}\right)=|\mathcal{C}| .
\end{aligned}
$$

Lemma 2.2. Let $m$ be a positive integer, $r \geq 3, k \in[r]$, and $m \geq k$. Let $y_{1}, y_{2}, \ldots, y_{k} \in \mathbb{N}_{0}$ and

$$
\begin{aligned}
\mathcal{C} & =\left\{\mathbf{u} \in P(m, r): \mathbf{u}(i) \neq y_{i} \text { for all } i \in[k-1] \text { and } \mathbf{u}(k)=0\right\}, \\
\mathcal{D} & =\left\{\mathbf{u} \in P(m, r): \mathbf{u}(i) \neq y_{i} \text { for all } i \in[k-1] \text { and } \mathbf{u}(k)=y_{k}\right\} .
\end{aligned}
$$

Then

(a) $\mathcal{D}=\mathcal{C}$ if $y_{k}=0$;

(b) $|\mathcal{D}|<|\mathcal{C}|$ if $k \in[r-1]$ and $y_{k}>0$;

(c) $|\mathcal{D}| \leq|\mathcal{C}|$ if $k=r$ and $y_{r}>0, y_{i}=0$ for some $1 \leq i \leq r-1$.

Proof. Clearly, part (a) is true. Suppose $y_{k}>0$. If $k=1$, then the result follows from Lemma 2.1. Let $k \geq 2$. Let $S_{i}=[m] \backslash\left\{y_{i}\right\}$ and set $S=S_{1} \times S_{2} \times$ $\cdots \times S_{k-1}$. For each $\left(d_{1}, \ldots, d_{k-1}\right) \in S$, let

$\mathcal{C}\left(d_{1}, \ldots, d_{k-1}\right)=\left\{\mathbf{u} \in P(m, r): \mathbf{u}(i)=d_{i}\right.$ for all $i \in[k-1]$ and $\left.\mathbf{u}(k)=0\right\}$, $\mathcal{D}\left(d_{1}, \ldots, d_{k-1}\right)=\left\{\mathbf{u} \in P(m, r): \mathbf{u}(i)=d_{i}\right.$ for all $i \in[k-1]$ and $\left.\mathbf{u}(k)=y_{k}\right\}$.

Note that

$$
\begin{aligned}
\mathcal{C} & =\bigcup_{\left(d_{1}, \ldots, d_{k-1}\right) \in S} \mathcal{C}\left(d_{1}, \ldots, d_{k-1}\right), \\
\mathcal{D} & =\bigcup_{\left(d_{1}, \ldots, d_{k-1}\right) \in S} \mathcal{D}\left(d_{1}, \ldots, d_{k-1}\right),
\end{aligned}
$$

Furthermore,

$$
\mathcal{C}\left(d_{1}, \ldots, d_{k-1}\right) \cap \mathcal{C}\left(d_{1}^{\prime}, \ldots, d_{k-1}^{\prime}\right)=\varnothing=\mathcal{D}\left(d_{1}, \ldots, d_{k-1}\right) \cap \mathcal{D}\left(d_{1}^{\prime}, \ldots, d_{k-1}^{\prime}\right)
$$

for all $\left(d_{1}, \ldots, d_{k-1}\right) \neq\left(d_{1}^{\prime}, \ldots, d_{k-1}^{\prime}\right)$. 
Suppose $k \in[r-2]$. By Lemma 2.1, $\left|\mathcal{D}\left(d_{1}, \ldots, d_{k-1}\right)\right| \leq\left|\mathcal{C}\left(d_{1}, \ldots, d_{k-1}\right)\right|$ for all $\left(d_{1}, \ldots, d_{k-1}\right) \in S$. It remains to show that

$$
\left|\mathcal{D}\left(d_{1}, \ldots, d_{k-1}\right)\right|<\left|\mathcal{C}\left(d_{1}, \ldots, d_{k-1}\right)\right|
$$

for at least one $\left(d_{1}, \ldots, d_{k-1}\right) \in S$. For each $i \in[k-1]$, set $z_{i}=0$ if $y_{i} \neq 0$ and $z_{i}=1$ if $y_{i}=0$. Then $\left(z_{1}, \ldots, z_{k-1}\right) \in S$. Since $m-\sum_{i=1}^{k-1} z_{i} \geq m-(k-1)>0$, by part (b) of Lemma $2.1,\left|\mathcal{D}\left(z_{1}, \ldots, z_{k-1}\right)\right|<\left|\mathcal{C}\left(z_{1}, \ldots, z_{k-1}\right)\right|$.

Suppose $k=r-1$. If $m-\sum_{i=1}^{r-2} d_{i}<0$, then

$$
\mathcal{D}\left(d_{1}, \ldots, d_{r-2}\right)=\mathcal{C}\left(d_{1}, \ldots, d_{r-2}\right)=\varnothing .
$$

If $m-\sum_{i=1}^{r-2} d_{i}=0$, then $\left|\mathcal{C}\left(d_{1}, \ldots, d_{r-2}\right)\right|=1$ and $\mathcal{D}\left(d_{1}, \ldots, d_{r-2}\right)=\varnothing$. If $m-\sum_{i=1}^{r-2} d_{i}>0$, then $\left|\mathcal{C}\left(d_{1}, \ldots, d_{r-2}\right)\right|=1$ and $\left|\mathcal{D}\left(d_{1}, \ldots, d_{r-2}\right)\right| \leq 1$. So, it remains to show that $m-\sum_{i=1}^{r-2} d_{i}=0$ for at least one $\left(d_{1}, \ldots, d_{k-1}\right) \in S$.

Note that $m \geq k \geq 2$. Suppose $y_{i} \neq 0$ for all $i \in[r-2]$. If $y_{i_{0}} \neq m$ for some $i_{0} \in[r-2]$, then set $d_{i}=0$ for all $i \in[r-2] \backslash\left\{i_{0}\right\}$ and $d_{i_{0}}=m$. If $y_{i}=m$ for all $i \in[r-2]$, then set $d_{1}=1, d_{2}=m-1$, and $d_{i}=0$ for all $i \in[r-2] \backslash\{1,2\}$. Let $U \subseteq[r-2]$ and $U \neq \varnothing$. Suppose that $y_{i}=0$ for all $i \in U$ and $y_{i} \neq 0$ for all $i \in[r-2] \backslash U$. Set $d_{i}=0$ for all $i \in[r-2] \backslash U$. Let $a \in U$. Set $d_{i}=1$ for all $i \in U \backslash\{a\}$ and $d_{a}=m-\sum_{i \in U \backslash\{a\}} d_{i} \geq m-(r-3)>0$. In either case, $m-\sum_{i=1}^{r-2} d_{i}=0$ and $\left(d_{1}, \ldots, d_{k-1}\right) \in S$. This concludes the proof of part (b).

The preceding argument does not apply to the case $k=r$. Instead, we will prove part (c) directly. Let $\left(d_{1}, \ldots, d_{r-1}, y_{r}\right) \in \mathcal{D}$. It is easy to see that the $\operatorname{map}\left(d_{1}, \ldots, d_{r-1}, y_{r}\right) \mapsto\left(d_{1}, \ldots, d_{i-1}, d_{i}+y_{r}, d_{i+1}, \ldots, d_{r-1}, 0\right)$ is an injection from $\mathcal{D}$ to $\mathcal{C}$. Thus, $|\mathcal{D}| \leq|\mathcal{C}|$.

Observe that the inequality in part (c) of the preceding lemma may not be strict. For example, taking $m=5, k=r=3$ and $\left(y_{1}, y_{2}, y_{3}\right)=(4,0,1)$, we have $\mathcal{C}=\{(2,3,0),(3,2,0),(0,5,0),(1,4,0)\}$ and $\mathcal{D}=\{(2,2,1),(3,1,1),(1,3,1)$, $(0,4,1)\}$. Furthermore, the condition that $y_{i}=0$ for some $1 \leq i \leq r-1$ is necessary. For example, taking $m=k=r=3$ and $\left(y_{1}, y_{2}, y_{3}\right)=(3,3,1)$, we have $\mathcal{C}=\{(2,1,0),(1,2,0)\}$ and $\mathcal{D}=\{(1,1,1),(2,0,1),(0,2,1)\}$.

Lemma 2.3. Let $m$ be a positive integer, $r \geq 3, k \in[r], k_{0} \in[k]$, and $m \geq k$. Let $y_{1}, y_{2}, \ldots, y_{k} \in \mathbb{N}_{0}$ and

$$
\begin{aligned}
\mathcal{C} & =\left\{\mathbf{u} \in P(m, r): \mathbf{u}(i)=y_{i} \text { for some } i \in[k] \backslash\left\{k_{0}\right\} \text { or } \mathbf{u}\left(k_{0}\right)=0\right\}, \\
\mathcal{D} & =\left\{\mathbf{u} \in P(m, r): \mathbf{u}(i)=y_{i} \text { for some } i \in[k]\right\} .
\end{aligned}
$$

Then

(a) $\mathcal{D}=\mathcal{C}$ if $y_{k_{0}}=0$;

(b) $|\mathcal{D}|<|\mathcal{C}|$ if $k \in[r-1]$ and $y_{k_{0}}>0$;

(c) $|\mathcal{D}| \leq|\mathcal{C}|$ if $k=r$ and $y_{k_{0}}>0, y_{i}=0$ for some $i \in[k] \backslash k_{0}$.

Proof. By relabelling if necessary, we may assume that $k_{0}=k$. Let

$$
\mathcal{E}_{0}=\left\{\mathbf{u} \in P(m, r): \mathbf{u}(i)=y_{i} \text { for some } i \in[k-1]\right\},
$$




$$
\begin{aligned}
\mathcal{C}_{1} & =\left\{\mathbf{u} \in P(m, r): \mathbf{u}(i) \neq y_{i} \text { for all } i \in[k-1] \text { and } \mathbf{u}(k)=0\right\}, \\
\mathcal{D}_{1} & =\left\{\mathbf{u} \in P(m, r): \mathbf{u}(i) \neq y_{i} \text { for all } i \in[k-1] \text { and } \mathbf{u}(k)=y_{k}\right\} .
\end{aligned}
$$

Then $\mathcal{C}=\mathcal{E}_{0} \cup \mathcal{C}_{1}$ and $\mathcal{D}=\mathcal{E}_{0} \cup \mathcal{D}_{1}$. Note that $\mathcal{E}_{0} \cap \mathcal{C}_{1}=\varnothing=\mathcal{E}_{0} \cap \mathcal{D}_{1}$. The result now follows from Lemma 2.2.

Lemma 2.4. Let $y_{1}, y_{2}, \ldots, y_{r} \in \mathbb{N}_{0}$ and $m$ be a positive integer. Let $r \geq 3$ and

$\mathcal{C}=\left\{\mathbf{u} \in P(m, r): \mathbf{u}(i)=y_{i}\right.$ for some $i \in[r-2]$ or $\mathbf{u}(r-1)=0$ or $\left.\mathbf{u}(r)=0\right\}$, $\mathcal{D}=\left\{\mathbf{u} \in P(m, r): \mathbf{u}(i)=y_{i}\right.$ for some $\left.i \in[r]\right\}$.

Then $|\mathcal{D}| \leq|\mathcal{C}|$.

Proof. If one of $y_{r-1}$ and $y_{r}$ is 0 , then the result follows immediately from part (a) or (c) of Lemma 2.3. So we may assume that $y_{r-1}>0$ and $y_{r}>0$. Let

$$
\begin{aligned}
& \mathcal{C}^{\prime}=\left\{\mathbf{u} \in P(m, r): \mathbf{u}(i)=y_{i} \text { for some } i \in[r-2] \text { or } \mathbf{u}(r-1)=0\right\} \\
& \mathcal{C}^{\prime \prime}=\{\mathbf{u} \in P(m, r): \mathbf{u}(i) \neq y_{i} \text { for all } i \in[r-2] \text { and } \mathbf{u}(r-1) \neq 0 \\
&\text { and } \mathbf{u}(r)=0\} \\
& \mathcal{D}^{\prime}=\{\mathbf{u} \in P(m, r):\left.\mathbf{u}(i)=y_{i} \text { for some } i \in[r-1]\right\} \\
& \mathcal{D}^{\prime \prime}=\{\mathbf{u} \in P(m, r): \mathbf{u}(i) \neq y_{i} \text { for all } i \in[r-2] \text { and } \mathbf{u}(r-1) \neq y_{r-1} \\
&\text { and } \left.\mathbf{u}(r)=y_{r}\right\}
\end{aligned}
$$

Clearly, $\mathcal{C}=\mathcal{C}^{\prime} \cup \mathcal{C}^{\prime \prime}, \mathcal{D}=\mathcal{D}^{\prime} \cup \mathcal{D}^{\prime \prime}$ and $\mathcal{C}^{\prime} \cap \mathcal{C}^{\prime \prime}=\varnothing=\mathcal{D}^{\prime} \cap \mathcal{D}^{\prime \prime}$. It follows from part (b) of Lemma 2.3 that $\left|\mathcal{D}^{\prime}\right|<\left|\mathcal{C}^{\prime}\right|$. On the other hand, the map $\left(d_{1}, \ldots, d_{r-1}, y_{r}\right) \mapsto\left(d_{1}, \ldots, d_{r-2}, d_{r-1}+y_{r}, 0\right)$ is an injection from $\mathcal{D}^{\prime \prime}$ to $\mathcal{C}^{\prime \prime}$. Hence, $|\mathcal{C}| \leq|\mathcal{D}|$.

Theorem 2.5. Let $y_{1}, \ldots, y_{r} \in \mathbb{N}_{0}$ and $r \geq 3$. Let $S \subseteq[r], S \neq \varnothing, m \geq|S|$, and

$$
\begin{aligned}
\mathcal{C} & =\{\mathbf{u} \in P(m, r): \mathbf{u}(i)=0 \text { for some } i \in S\} \\
\mathcal{D} & =\left\{\mathbf{u} \in P(m, r): \mathbf{u}(i)=y_{i} \text { for some } i \in S\right\} .
\end{aligned}
$$

If $\sum_{s \in S} y_{s}>0$, then $|\mathcal{D}|<|\mathcal{C}|$.

Proof. Without loss of generality, we may assume that $S=[k]$ for some $k \in[r]$. By relabelling if necessary, we may assume that $y_{1} \geq y_{2} \geq \cdots \geq y_{k}$. Since $\sum_{j=1}^{k} y_{j}>0$, we have $y_{1}>0$. If $k=1$, then by Lemma 2.1, the theorem holds. Suppose $k \geq 2$.

We shall distinguish two cases.

Case 1. Suppose $k \in[r-1]$. For each $j \in[k-1]$, let

$$
\begin{aligned}
\mathcal{F}_{j}=\{\mathbf{u} \in P(m, r): & \mathbf{u}(i)=y_{i} \text { for some } i \in[j] \text { or } \\
\mathbf{u}(i) & =0 \text { for some } j+1 \leq i \leq k\} .
\end{aligned}
$$


By Lemma 2.3, $\left|\mathcal{F}_{1}\right|<|\mathcal{C}|$. Again, by Lemma 2.3,

$$
|\mathcal{D}| \leq\left|\mathcal{F}_{k-1}\right| \leq \cdots \leq\left|\mathcal{F}_{2}\right| \leq\left|\mathcal{F}_{1}\right|
$$

Hence, $|\mathcal{D}|<|\mathcal{C}|$.

Case 2. Suppose $k=r$. For each $j \in[r-2]$, let

$$
\begin{aligned}
\mathcal{F}_{j}=\{\mathbf{u} \in P(m, r): & \mathbf{u}(i)=y_{i} \text { for some } i \in[j] \text { or } \\
\mathbf{u}(i) & =0 \text { for some } j+1 \leq i \leq r\} .
\end{aligned}
$$

By Lemma 2.3, $\left|\mathcal{F}_{1}\right|<|\mathcal{C}|$. Again, by Lemma 2.3,

$$
\left|\mathcal{F}_{r-2}\right| \leq \cdots \leq\left|\mathcal{F}_{2}\right| \leq\left|\mathcal{F}_{1}\right|
$$

By Lemma 2.4, $|\mathcal{D}| \leq\left|\mathcal{F}_{r-2}\right|$. Hence, $|\mathcal{D}|<|\mathcal{C}|$.

Let $\mathbf{u}=\left(u_{1}, u_{2}, \ldots, u_{l}\right) \in P(n, l)$. We define $R(i, \mathbf{u})$ to be the element obtained from $\mathbf{u}$ by removing the $i$-th coordinate, i.e.,

$$
R(i ; \mathbf{u})=\left(u_{1}, u_{2}, \ldots, u_{i-1}, u_{i+1}, \ldots, u_{l}\right) .
$$

Inductively, if $x_{1}, x_{2}, \ldots, x_{t}$ are distinct elements in $[l]$ with $x_{1}<x_{2}<\cdots<x_{t}$, we define

$$
R\left(x_{1}, x_{2}, \ldots, x_{t} ; \mathbf{u}\right)=R\left(x_{1}, x_{2}, \ldots, x_{t-1} ; R\left(x_{t} ; \mathbf{u}\right)\right) .
$$

In other words, $R\left(x_{1}, x_{2}, \ldots, x_{t} ; \mathbf{u}\right)$ is the element obtained from $\mathbf{u}$ by removing the coordinates $x_{i}$.

Corollary 2.6. Let $w_{1}, \ldots, w_{t}, y_{t+1}, \ldots, y_{r+t} \in \mathbb{N}_{0}, t \geq 1$ and $r \geq 3$. Let $S \subseteq[r+t] \backslash[t], S \neq \varnothing, m \geq|S|+\sum_{1 \leq i \leq t} w_{i}$, and

$\mathcal{D}=\left\{\mathbf{u} \in P(m, r+t): \mathbf{u}(i)=w_{i}\right.$ for all $i \in[t]$ and $\mathbf{u}(i)=y_{i}$ for some $\left.i \in S\right\}$.

If $\sum_{s \in S} y_{s}>0$, then

$$
|\mathcal{D}|<\sum_{0 \leq d \leq|S|-1}\left(\begin{array}{c}
m-\sum_{1 \leq i \leq t} w_{i}-d+r-2 \\
r-2
\end{array}\right) .
$$

Proof. Let

$\mathcal{C}=\left\{\mathbf{u} \in P(m, r+t): \mathbf{u}(i)=w_{i}\right.$ for all $i \in[t]$ and $\mathbf{u}(i)=0$ for some $\left.i \in S\right\}$.

Let $S^{*}=\{a-t: a \in S\}, y_{i}^{*}=y_{i+t}$ for all $i \in S^{*}$,

$$
\begin{aligned}
& \mathcal{C}^{*}=\left\{\mathbf{u} \in P\left(m-\sum_{1 \leq i \leq t} w_{i}, r\right): \mathbf{u}(i)=0 \text { for some } i \in S^{*}\right\}, \\
& \mathcal{D}^{*}=\left\{\mathbf{u} \in P\left(m-\sum_{1 \leq i \leq t} w_{i}, r\right): \mathbf{u}(i)=y_{i}^{*} \text { for some } i \in S^{*}\right\} .
\end{aligned}
$$

By Theorem 2.5, $\left|\mathcal{D}^{*}\right|<\left|\mathcal{C}^{*}\right|$. For each $\mathbf{u} \in P(m, r+t)$, the mapping

$$
\mathbf{u} \rightarrow R(1, \ldots, t ; \mathbf{u})
$$


is a bijection from $\mathcal{C}$ onto $\mathcal{C}^{*}$ and from $\mathcal{D}$ onto $\mathcal{D}^{*}$. Thus, $|\mathcal{D}|<|\mathcal{C}|$.

Let

$$
\mathcal{F}=\left\{\mathbf{u} \in P(m, r+t): \mathbf{u}(i)=w_{i} \text { for all } i \in[t]\right\}
$$

Then

$$
\mathcal{C}=\mathcal{F} \backslash\{\mathbf{u} \in P(m, r+t): \mathbf{u}(i) \neq 0 \text { for all } i \in S\}
$$

and

$$
\begin{aligned}
|\mathcal{C}| & =\left(\begin{array}{c}
m-\sum_{1 \leq i \leq t} w_{i}+r-1 \\
r-1
\end{array}\right)-\left(\begin{array}{c}
m-\sum_{1 \leq i \leq t} w_{i}-|S|+r-1 \\
r-1
\end{array}\right) \\
& =\sum_{0 \leq d \leq|S|-1}\left(\begin{array}{c}
m-\sum_{1 \leq i \leq t} w_{i}-d+r-2 \\
r-2
\end{array}\right) .
\end{aligned}
$$

Hence, the corollary holds.

\section{Main result}

For any $\mathbf{u}, \mathbf{v} \in P(n, l)$, let $I(\mathbf{u}, \mathbf{v})=\{i: \mathbf{u}(i)=\mathbf{v}(i)\}$. A family $\mathcal{B} \subseteq P(n, l)$ is said to be independent if $I(\mathbf{u}, \mathbf{v})=\varnothing$, i.e., $|I(\mathbf{u}, \mathbf{v})|=0$, for all $\mathbf{u}, \mathbf{v} \in \mathcal{B}$ with $\mathbf{u} \neq \mathbf{v}$.

We shall need the following theorem [30, Theorem 2.3].

Theorem 3.1. Let $m, n$ be positive integers satisfying $m \leq n$, and let $q, r, s$ be positive integers with $r, s \geq 2$ and $n \geq(2 s)^{2^{r-2} q}+1$. If $\mathcal{A} \subseteq P(m, r)$ such that $|\mathcal{A}| \geq n^{\frac{1}{q}}\left(\begin{array}{c}n+r-2 \\ r-2\end{array}\right)$, then there is an independent set $\mathcal{B} \subseteq \mathcal{A}$ with $|\mathcal{B}| \geq s+1$.

Let $\mathcal{A} \subseteq P(n, l)$. Let $x_{1}, x_{2}, \ldots, x_{t}$ be distinct elements in [l] with $x_{1}<x_{2}<$ $\cdots<x_{t}$, and $y_{1}, y_{2}, \ldots, y_{t} \in \mathbb{N}_{0}$ with $\sum_{j=1}^{t} y_{j} \leq n$. We set

$$
\begin{aligned}
& \mathcal{A}\left(x_{1}, x_{2}, \ldots, x_{t} ; y_{1}, y_{2}, \ldots, y_{t}\right)=\left\{\mathbf{u} \in \mathcal{A}: \mathbf{u}\left(x_{i}\right)=y_{i} \text { for all } i\right\}, \\
& \mathcal{A}^{*}\left(x_{1}, x_{2}, \ldots, x_{t} ; y_{1}, y_{2}, \ldots, y_{t}\right) \\
= & \left\{R\left(x_{1}, x_{2}, \ldots, x_{t} ; \mathbf{u}\right): \mathbf{u} \in \mathcal{A}\left(x_{1}, x_{2}, \ldots, x_{t} ; y_{1}, y_{2}, \ldots, y_{t}\right)\right\} .
\end{aligned}
$$

Note that

$$
\mathcal{A}^{*}\left(x_{1}, x_{2}, \ldots, x_{t} ; y_{1}, y_{2}, \ldots, y_{t}\right) \subseteq P\left(n-\sum_{j=1}^{t} y_{j}, l-t\right),
$$

and

$$
\left|\mathcal{A}^{*}\left(x_{1}, x_{2}, \ldots, x_{t} ; y_{1}, y_{2}, \ldots, y_{t}\right)\right|=\left|\mathcal{A}\left(x_{1}, x_{2}, \ldots, x_{t} ; y_{1}, y_{2}, \ldots, y_{t}\right)\right| .
$$

Lemma 3.2. Let $\mathcal{A} \subseteq P(n, l)$ be t-intersecting and $l \geq t+3$. Let $x_{1}, x_{2}, \ldots$, $x_{t+1}$ be distinct elements in $[l]$ with $x_{1}<x_{2}<\cdots<x_{t+1}$, and $y_{1}, y_{2}, \ldots, y_{t+1} \in$ $\mathbb{N}_{0}$ with $\sum_{j=1}^{t+1} y_{j} \leq n$. If $\mathcal{A}^{*}\left(x_{1}, \ldots, x_{t+1} ; y_{1}, \ldots, y_{t+1}\right)$ has an independent set of size at least $l-t$, then

$$
\mathcal{A} \subseteq \bigcup_{\substack{T \subseteq[t+1],|T|=t}}\left\{\mathbf{u} \in P(n, l): \mathbf{u}\left(x_{s}\right)=y_{s} \text { for all } s \in T\right\} .
$$


Proof. Let

$$
\mathcal{B}=\left\{R\left(x_{1}, x_{2}, \ldots, x_{t+1} ; \mathbf{u}_{i}\right): i=1,2, \ldots, l-t\right\}
$$

be an independent set of size $l-t$ in $\mathcal{A}^{*}\left(x_{1}, \ldots, x_{t+1} ; y_{1}, \ldots, y_{t+1}\right)$. Here each $\mathbf{u}_{i}$ is an element of $\mathcal{A}$ such that $\mathbf{u}_{i}\left(x_{j}\right)=y_{j}$ for all $1 \leq j \leq t$.

Let $\mathbf{v} \in \mathcal{A}$. Suppose

$$
\mathbf{v} \notin \bigcup_{\substack{T \subseteq[t+1],|T|=t}}\left\{\mathbf{u} \in P(n, l): \mathbf{u}\left(x_{s}\right)=y_{s} \text { for all } s \in T\right\} .
$$

Then there exist $j_{1}$ and $j_{2}$ with $1 \leq j_{1}<j_{2} \leq t+1$ such that $\mathbf{v}\left(x_{j_{1}}\right) \neq y_{j_{1}}$ and $\mathbf{v}\left(x_{j_{2}}\right) \neq y_{j_{2}}$. Since $\mathcal{A}$ is $t$-intersecting,

$$
\left|I\left(R\left(x_{1}, x_{2}, \ldots, x_{t+1} ; \mathbf{v}\right), R\left(x_{1}, x_{2}, \ldots, x_{t+1} ; \mathbf{u}_{i}\right)\right)\right| \geq 1
$$

for $i=1,2, \ldots, l-t$.

Set $\mathbf{z}=R\left(x_{1}, x_{2}, \ldots, x_{t+1} ; \mathbf{v}\right)$ and $\mathbf{w}_{i}=R\left(x_{1}, x_{2}, \ldots, x_{t+1} ; \mathbf{u}_{i}\right)$. Since $\mathcal{B}$ is independent,

$$
I\left(\mathbf{z}, \mathbf{w}_{i}\right) \cap I\left(\mathbf{z}, \mathbf{w}_{i^{\prime}}\right)=\varnothing
$$

for $i \neq i^{\prime}$. Therefore $\left|\bigcup_{i=1}^{l-t} I\left(\mathbf{z}, \mathbf{w}_{i}\right)\right|=\sum_{i=1}^{l-t}\left|I\left(\mathbf{z}, \mathbf{w}_{i}\right)\right| \geq \sum_{i=1}^{l-t} 1=l-t$, but on the other hand, $\bigcup_{i=1}^{l-t} I\left(\mathbf{z}, \mathbf{w}_{i}\right) \subseteq[l] \backslash\left\{x_{j}: j \in[t+1]\right\}$ which is of size at most $l-t-1$, a contradiction. Hence,

$$
\mathbf{v} \in \bigcup_{\substack{T \subseteq[t+1],|T|=t}}\left\{\mathbf{u} \in P(n, l): \mathbf{u}\left(x_{s}\right)=y_{s} \text { for all } s \in T\right\},
$$

and the lemma follows.

Lemma 3.3. If $x_{1}, x_{2}, \ldots, x_{r}$ are positive real numbers, then

$$
\prod_{i=1}^{r}\left(1+x_{i}\right)-\prod_{i=1}^{r}\left(1-x_{r}\right) \geq 2 \sum_{i=1}^{r} x_{i}
$$

Proof. Let $S \subseteq[r]$. If $|S|$ is even, then $\prod_{s \in S} x_{s}$ appears in the expansions of $\prod_{i=1}^{r}\left(1+x_{i}\right)$ and $\prod_{i=1}^{r}\left(1-x_{r}\right)$. So, $\prod_{s \in S} x_{s}$ does not appear in $\prod_{i=1}^{r}(1+$ $\left.x_{i}\right)-\prod_{i=1}^{r}\left(1-x_{r}\right)$. If $|S|$ is odd, then $\prod_{s \in S} x_{s}$ and $-\prod_{s \in S} x_{s}$ appear in the expansions of $\prod_{i=1}^{r}\left(1+x_{i}\right)$ and $\prod_{i=1}^{r}\left(1-x_{r}\right)$, respectively. So, $2 \prod_{s \in S} x_{s}$ appears in $\prod_{i=1}^{r}\left(1+x_{i}\right)-\prod_{i=1}^{r}\left(1-x_{r}\right)$. The lemma follows by just considering all subsets $S$ of $[r]$ with $|S|=1$.

Lemma 3.4. Let $m, n$ be positive integers with $n \geq m+1$. Then

$$
\left(\begin{array}{c}
n+m \\
m
\end{array}\right)-\left(\begin{array}{c}
n-1 \\
m
\end{array}\right) \geq \frac{(m+1) n^{m-1}}{(m-1) !}
$$

Proof. Note that

$$
\left(\begin{array}{c}
n+m \\
m
\end{array}\right)=\frac{n^{m}}{m !} \prod_{i=1}^{m}\left(1+\frac{i}{n}\right)
$$


and

It then follows from Lemma 3.3 that

$$
\left(\begin{array}{c}
n-1 \\
m
\end{array}\right)=\frac{n^{m}}{m !} \prod_{i=1}^{m}\left(1-\frac{i}{n}\right)
$$

$$
\left(\begin{array}{c}
n+m \\
m
\end{array}\right)-\left(\begin{array}{c}
n-1 \\
m
\end{array}\right) \geq \frac{n^{m}}{m !}\left(2 \sum_{i=1}^{m} \frac{i}{n}\right)=\frac{(m+1) n^{m-1}}{(m-1) !}
$$

Lemma 3.5. Let $m, n$ be positive integers with $n \geq m$. Then

$$
\frac{n^{m}}{m !}<\left(\begin{array}{c}
n+m \\
m
\end{array}\right)<\frac{n^{m}}{m !}\left(1+\frac{2^{m} m}{n}\right) .
$$

Proof. Note that

$$
\left(\begin{array}{c}
n+m \\
m
\end{array}\right)=\frac{n^{m}}{m !} \prod_{i=1}^{m}\left(1+\frac{i}{n}\right)>\frac{n^{m}}{m !} .
$$

For the second inequality, it is sufficient to show that

$$
\prod_{i=1}^{m}\left(1+\frac{i}{n}\right)<\left(1+\frac{2^{m} m}{n}\right) \text {. }
$$

Now,

$$
\begin{aligned}
\prod_{i=1}^{m}\left(1+\frac{i}{n}\right) & \leq\left(1+\frac{m}{n}\right)^{m} \\
& =\left(1+\sum_{i=1}^{m}\left(\begin{array}{c}
m \\
i
\end{array}\right)\left(\frac{m}{n}\right)^{i}\right) \\
& \leq\left(1+\frac{m}{n}\left(\sum_{i=1}^{m}\left(\begin{array}{c}
m \\
i
\end{array}\right)\right)\right) \\
& <\left(1+\frac{2^{m} m}{n}\right) .
\end{aligned}
$$

Lemma 3.6. Let $m$ be a positive integer and $f, g$ be positive real numbers. There exists a constant $n_{0}=n_{0}(f, g, m)$ depending on $f, g$ and $m$ such that if $n \geq n_{0}$, then

$$
f\left(\begin{array}{c}
n+m \\
m
\end{array}\right)+g \sqrt{n}\left(\begin{array}{c}
n+m-1 \\
m-1
\end{array}\right)<\frac{n^{m}(f+1)}{m !} .
$$

Proof. Suppose $n>\max \left(m, 2^{m-1}(m-1),\left(\frac{2^{m} f}{g}\right)^{2},(3 g m)^{2}\right)$. By Lemma 3.5,

$$
f\left(\begin{array}{c}
n+m \\
m
\end{array}\right)+g \sqrt{n}\left(\begin{array}{c}
n+m-1 \\
m-1
\end{array}\right)
$$




$$
\begin{aligned}
& <f \frac{n^{m}}{m !}\left(1+\frac{2^{m} m}{n}\right)+g \sqrt{n} \frac{n^{m-1}}{(m-1) !}\left(1+\frac{2^{m-1}(m-1)}{n}\right) \\
& \leq f \frac{n^{m}}{m !}\left(1+\frac{2^{m} m}{n}\right)+\frac{2 g n^{m-\frac{1}{2}}}{(m-1) !} \\
& =f \frac{n^{m}}{m !}\left(1+\frac{2^{m} m}{n}+\frac{2 g m}{f \sqrt{n}}\right) \\
& \leq f \frac{n^{m}}{m !}\left(1+\frac{3 g m}{f \sqrt{n}}\right) \\
& =\frac{n^{m}}{m !}\left(f+\frac{3 g m}{\sqrt{n}}\right) \\
& <\frac{n^{m}(f+1)}{m !} .
\end{aligned}
$$

Proof of Theorem 1.3. We may assume that $\mathcal{A}$ is maximally $t$-intersecting in the sense that $\mathcal{A} \cup\{\mathbf{u}\}$ is not $t$-intersecting for any $\mathbf{u} \in P(n, l) \backslash \mathcal{A}$.

Suppose

$$
|\mathcal{A}|<\frac{n^{l-t-2}(t+3)}{(l-t-2) !}
$$

By Lemma 3.4,

$$
\left(\begin{array}{c}
n+l-t-1 \\
l-t-1
\end{array}\right)-\left(\begin{array}{c}
n-1 \\
l-t-1
\end{array}\right) \geq \frac{(l-t) n^{l-t-2}}{(l-t-2) !}
$$

Since $l \geq 2 t+3,|\mathcal{A}|<\left(\begin{array}{c}n+l-t-1 \\ l-t-1\end{array}\right)-\left(\begin{array}{c}n-1 \\ l-t-1\end{array}\right)$. So, we may assume that

$$
|\mathcal{A}| \geq \frac{n^{l-t-2}(t+3)}{(l-t-2) !} .
$$

Let $\mathbf{w}=\left(w_{1}, w_{2}, \ldots, w_{l}\right) \in \mathcal{A}$ be fixed. Then

$$
\mathcal{A}=\bigcup_{\substack{\left\{x_{1}, x_{2}, \ldots, x_{t}\right\} \subseteq[l], x_{1}<x_{2}<\cdots<x_{t}}} \mathcal{A}\left(x_{1}, x_{2}, \ldots, x_{t} ; w_{x_{1}}, w_{x_{2}}, \ldots, w_{x_{t}}\right) .
$$

Let $\left\{x_{1}^{\prime}, x_{2}^{\prime}, \ldots, x_{t}^{\prime}\right\} \subseteq[l]$ with $x_{1}^{\prime}<x_{2}^{\prime}<\cdots<x_{t}^{\prime}$ be fixed and

$$
\mathcal{C}=\mathcal{A}\left(x_{1}^{\prime}, x_{2}^{\prime}, \ldots, x_{t}^{\prime} ; w_{x_{1}^{\prime}}, w_{x_{2}^{\prime}}, \ldots, w_{x_{t}^{\prime}}\right) .
$$

We may assume that $|\mathcal{C}|$ is maximum in the sense that

$$
\left|\mathcal{A}\left(x_{1}, x_{2}, \ldots, x_{t} ; w_{x_{1}}, w_{x_{2}}, \ldots, w_{x_{t}}\right)\right| \leq|\mathcal{C}|
$$

for all $\left\{x_{1}, x_{2}, \ldots, x_{t}\right\} \subseteq[l]$ with $x_{1}<x_{2}<\cdots<x_{t}$.

By relabelling if necessary, we may assume that $x_{i}^{\prime}=i$ for all $i$, i.e.,

$$
\mathcal{C}=\mathcal{A}\left(1,2, \ldots, t ; w_{1}, w_{2}, \ldots, w_{t}\right) .
$$

Let $P=P(n, l)$. Since $\mathcal{A}$ is non-trivially $t$-intersecting,

$$
\mathcal{A} \nsubseteq P\left(1,2, \ldots, t ; w_{1}, w_{2}, \ldots, w_{t}\right) .
$$


Therefore there is a $\mathbf{y}=\left(y_{1}, y_{2}, \ldots, y_{l}\right) \in \mathcal{A}$ with $y_{i} \neq w_{i}$ for some $1 \leq i \leq t$. Since $\mathcal{C} \cup\{\mathbf{y}\}$ is $t$-intersecting,

$$
\mathcal{C}=\bigcup_{t+1 \leq s \leq l} \mathcal{A}\left(1,2, \ldots, t, s ; w_{1}, w_{2}, \ldots, w_{t}, y_{s}\right) .
$$

We shall distinguish 5 cases.

Case 1. Suppose that

$$
\left|\mathcal{A}\left(1,2, \ldots, t, s ; w_{1}, w_{2}, \ldots, w_{t}, y_{s}\right)\right| \leq n^{\frac{1}{2}}\left(\begin{array}{c}
n+l-t-3 \\
l-t-3
\end{array}\right)
$$

for all $t+1 \leq s \leq l$. Then

$$
|\mathcal{C}| \leq(l-t) n^{\frac{1}{2}}\left(\begin{array}{c}
n+l-t-3 \\
l-t-3
\end{array}\right) .
$$

By the maximality of $|\mathcal{C}|$,

$$
|\mathcal{A}| \leq\left(\begin{array}{l}
l \\
t
\end{array}\right)(l-t) n^{\frac{1}{2}}\left(\begin{array}{c}
n+l-t-3 \\
l-t-3
\end{array}\right) .
$$

By Lemma 3.6,

$$
|\mathcal{A}|<\frac{n^{l-t-2}}{(l-t-2) !},
$$

contradicting equation (1). Hence, Case 1 cannot happen.

Now, by relabelling if necessary we may assume that there is a $k \in\{t+1$, $t+2, \ldots, l\}$ such that

$$
\left|\mathcal{A}\left(1,2, \ldots, t, s ; w_{1}, w_{2}, \ldots, w_{t}, y_{s}\right)\right| \geq n^{\frac{1}{2}}\left(\begin{array}{c}
n+l-t-3 \\
l-t-3
\end{array}\right)
$$

for all $t+1 \leq s \leq k$ and

$$
\left|\mathcal{A}\left(1,2, \ldots, t, s ; w_{1}, w_{2}, \ldots, w_{t}, y_{s}\right)\right| \leq n^{\frac{1}{2}}\left(\begin{array}{c}
n+l-t-3 \\
l-t-3
\end{array}\right)
$$

for all $k+1 \leq s \leq l$. Note that if $k=l$, then there will be no family satisfying the preceding inequality.

Case 2. Suppose $k=t+1$. Since

$$
\begin{aligned}
& \left|\mathcal{A}^{*}\left(1,2, \ldots, t, t+1 ; w_{1}, w_{2}, \ldots, w_{t}, y_{t+1}\right)\right| \\
= & \left|\mathcal{A}\left(1,2, \ldots, t, t+1 ; w_{1}, w_{2}, \ldots, w_{t}, y_{t+1}\right)\right|,
\end{aligned}
$$

and

$$
\mathcal{A}^{*}\left(1,2, \ldots, t, t+1 ; w_{1}, w_{2}, \ldots, w_{t}, y_{t+1}\right) \subseteq P\left(n-y_{t+1}-\sum_{j=1}^{t} w_{j}, l-t-1\right),
$$


by Theorem $3.1, \mathcal{A}^{*}\left(1,2, \ldots, t, t+1 ; w_{1}, w_{2}, \ldots, w_{t}, y_{t+1}\right)$ has an independent set of size at least $l-t$, if $n \geq(2(l-t-1))^{2^{l-t-2}}+1$. Then it follows from Lemma 3.2 that

$\mathcal{A}=\mathcal{C} \cup \bigcup_{1 \leq j \leq t} \mathcal{A}\left(1, \ldots, j-1, j+1, \ldots, t, t+1 ; w_{1}, \ldots, w_{j-1}, w_{j+1}, \ldots, w_{t}, y_{t+1}\right)$.

By the choice of $|\mathcal{C}|,|\mathcal{A}| \leq(t+1)|\mathcal{C}|$.

Now,

$$
\begin{aligned}
& \left|\mathcal{A}\left(1,2, \ldots, t, t+1 ; w_{1}, w_{2}, \ldots, w_{t}, y_{t+1}\right)\right| \\
\leq & \left(\begin{array}{c}
n-y_{t+1}-\left(\sum_{j=1}^{t} w_{j}\right)+l-t-2 \\
l-t-2
\end{array}\right) \\
\leq & \left(\begin{array}{c}
n+l-t-2 \\
l-t-2
\end{array}\right) .
\end{aligned}
$$

Therefore

$$
|\mathcal{C}| \leq\left(\begin{array}{c}
n+l-t-2 \\
l-t-2
\end{array}\right)+(l-t-1) n^{\frac{1}{2}}\left(\begin{array}{c}
n+l-t-3 \\
l-t-3
\end{array}\right)
$$

and

$$
|\mathcal{A}| \leq(t+1)\left(\begin{array}{c}
n+l-t-2 \\
l-t-2
\end{array}\right)+(t+1)(l-t-1) n^{\frac{1}{2}}\left(\begin{array}{c}
n+l-t-3 \\
l-t-3
\end{array}\right) .
$$

By Lemma 3.6,

$$
|\mathcal{A}|<\frac{n^{l-t-2}(t+2)}{(l-t-2) !}
$$

contradicting equation (1). Hence, Case 2 cannot happen.

Case 3. Suppose $k=t+2$. Again, by Theorem 3.1, $\mathcal{A}^{*}\left(1,2, \ldots, t, s ; w_{1}, w_{2}, \ldots\right.$, $\left.w_{t}, y_{s}\right)$ has an independent set of size at least $l-t$ for $s \in\{t+1, t+2\}$, if $n \geq(2(l-t-1))^{2^{l-t-2}}+1$. For each $j \in[t]$, let

$$
\begin{gathered}
\mathcal{Q}_{j}=\left\{\mathbf{u} \in \mathcal{A}: \mathbf{u}(i)=w_{i} \text { for all } i \in[t] \backslash\{j\}, \mathbf{u}(j) \neq w_{j},\right. \\
\left.\mathbf{u}(t+1)=y_{t+1} \text { and } \mathbf{u}(t+2)=y_{t+2}\right\} .
\end{gathered}
$$

It follows from Lemma 3.2 that

$$
\mathcal{A}=\mathcal{C} \cup \bigcup_{1 \leq j \leq t} \mathcal{Q}_{j}
$$

Let $n_{j}^{\prime}=n-y_{t+1}-y_{t+2}-\left(\sum_{1 \leq i \leq t, i \neq j} w_{i}\right)$. If $n_{j}^{\prime}<0$, then $\left|\mathcal{Q}_{j}\right|=0$. If $n_{j}^{\prime} \geq 0$, then

$$
\left|\mathcal{Q}_{j}\right| \leq \sum_{\substack{0 \leq d \leq n_{j}^{\prime}, d \neq w_{j}}}\left(\begin{array}{c}
n_{j}^{\prime}-d+l-t-3 \\
l-t-3
\end{array}\right)
$$




$$
\begin{aligned}
& \leq \sum_{0 \leq d \leq n_{j}^{\prime}}\left(\begin{array}{c}
n_{j}^{\prime}-d+l-t-3 \\
l-t-3
\end{array}\right) \\
& =\left(\begin{array}{c}
n_{j}^{\prime}+l-t-2 \\
l-t-2
\end{array}\right) \\
& \leq\left(\begin{array}{c}
n+l-t-2 \\
l-t-2
\end{array}\right) .
\end{aligned}
$$

Therefore $|\mathcal{A}| \leq|\mathcal{C}|+t\left(\begin{array}{c}n+l-t-2 \\ l-t-2\end{array}\right)$.

Now, for each $s \in\{t+1, t+2\}$,

$$
\begin{aligned}
\left|\mathcal{A}\left(1,2, \ldots, t, s ; w_{1}, w_{2}, \ldots, w_{t}, y_{s}\right)\right| & \leq\left(\begin{array}{c}
n-y_{s}-\left(\sum_{j=1}^{t} w_{j}\right)+l-t-2 \\
l-t-2
\end{array}\right) \\
& \leq\left(\begin{array}{c}
n+l-t-2 \\
l-t-2
\end{array}\right) .
\end{aligned}
$$

Therefore

$$
|\mathcal{C}| \leq 2\left(\begin{array}{c}
n+l-t-2 \\
l-t-2
\end{array}\right)+(l-t-2) n^{\frac{1}{2}}\left(\begin{array}{c}
n+l-t-3 \\
l-t-3
\end{array}\right),
$$

and

$$
|\mathcal{A}| \leq(t+2)\left(\begin{array}{c}
n+l-t-2 \\
l-t-2
\end{array}\right)+(l-t-2) n^{\frac{1}{2}}\left(\begin{array}{c}
n+l-t-3 \\
l-t-3
\end{array}\right) .
$$

By Lemma 3.6,

$$
|\mathcal{A}|<\frac{n^{l-t-2}(t+3)}{(l-t-2) !}
$$

contradicting equation (1). Hence, Case 3 cannot happen.

We may assume that $t+3 \leq k \leq l$. In particular,

$$
\left|\mathcal{A}\left(1,2, \ldots, t, t+1 ; w_{1}, w_{2}, \ldots, w_{t}, y_{t+1}\right)\right| \geq n^{\frac{1}{2}}\left(\begin{array}{c}
n+l-t-3 \\
l-t-3
\end{array}\right) .
$$

On the other hand,

$$
\begin{aligned}
& \left|\mathcal{A}\left(1,2, \ldots, t, t+1 ; w_{1}, w_{2}, \ldots, w_{t}, y_{t+1}\right)\right| \\
\leq & \left(\begin{array}{c}
n-y_{t+1}-\left(\sum_{j=1}^{t} w_{j}\right)+l-t-2 \\
l-t-2
\end{array}\right) \\
\leq & \left(\begin{array}{c}
n-\left(\sum_{j=1}^{t} w_{j}\right)+l-t-2 \\
l-t-2
\end{array}\right) .
\end{aligned}
$$

If $n-\sum_{j=1}^{t} w_{j}<l-t$, then $\left(\begin{array}{c}n-\left(\sum_{j=1}^{t} w_{j}\right)+l-t-2 \\ l-t-2\end{array}\right)<\left(\begin{array}{c}2 l-2 t-2 \\ l-t-2\end{array}\right)$, which is impossible for large $n$. So, we may assume that

$$
n-\sum_{j=1}^{t} w_{j} \geq l-t .
$$


Case 4. Suppose $t+3 \leq k \leq l-1$. Again, by Theorem $3.1, \mathcal{A}^{*}(1,2, \ldots, t, s$; $\left.w_{1}, w_{2}, \ldots, w_{t}, y_{s}\right)$ has an independent set of size at least $l-t$ for $s \in\{t+1$, $t+2, t+3\}$, if $n \geq(2(l-t-1))^{2^{l-t-2}}+1$. For each $j \in[t]$, let

$$
\begin{aligned}
\mathcal{Q}_{j}=\left\{\mathbf{u} \in \mathcal{A}: \mathbf{u}(i)=w_{i} \text { for all } i \in[t] \backslash\{j\}, \mathbf{u}(j) \neq w_{j},\right. \\
\left.\mathbf{u}(t+1)=y_{t+1}, \mathbf{u}(t+2)=y_{t+2}, \text { and } \mathbf{u}(t+3)=y_{t+3}\right\} .
\end{aligned}
$$

It follows from Lemma 3.2 that

$$
\mathcal{A}=\mathcal{C} \cup \bigcup_{1 \leq j \leq t} \mathcal{Q}_{j} .
$$

Let $n_{j}^{\prime}=n-y_{t+1}-y_{t+2}-y_{t+3}-\left(\sum_{1 \leq i \leq t, i \neq j} w_{i}\right)$. If $n_{j}^{\prime}<0$, then $\left|\mathcal{Q}_{j}\right|=0$. If $n_{j}^{\prime} \geq 0$, then

$$
\begin{aligned}
\left|\mathcal{Q}_{j}\right| & \leq \sum_{\substack{0 \leq d \leq n_{j}^{\prime} \\
d \neq w_{j}}}\left(\begin{array}{c}
n_{j}^{\prime}-d+l-t-4 \\
l-t-4
\end{array}\right) \\
& \leq \sum_{0 \leq d \leq n_{j}^{\prime}}\left(\begin{array}{c}
n_{j}^{\prime}-d+l-t-4 \\
l-t-4
\end{array}\right) \\
& =\left(\begin{array}{c}
n_{j}^{\prime}+l-t-3 \\
l-t-3
\end{array}\right) \\
& \leq\left(\begin{array}{c}
n+l-t-3 \\
l-t-3
\end{array}\right) .
\end{aligned}
$$

Therefore $|\mathcal{A}| \leq|\mathcal{C}|+t\left(\begin{array}{c}n+l-t-3 \\ l-t-3\end{array}\right)$.

Let

$$
\begin{aligned}
\mathcal{D}=\{\mathbf{u} \in P(n, l): \mathbf{u}(i) & =w_{i} \text { for all } i \in[t] \text { and } \\
\mathbf{u}(i) & \left.=y_{i} \text { for some } t+1 \leq i \leq l-1\right\} .
\end{aligned}
$$

By equation (2) and Corollary 2.6,

$$
\begin{aligned}
|\mathcal{D}| & \leq \sum_{0 \leq d \leq l-t-2}\left(\begin{array}{c}
n-\sum_{1 \leq i \leq t} w_{i}-d+l-t-2 \\
l-t-2
\end{array}\right) \\
& \leq \sum_{0 \leq d \leq l-t-2}\left(\begin{array}{c}
n-d+l-t-2 \\
l-t-2
\end{array}\right) .
\end{aligned}
$$

Since

we have

$$
\bigcup_{t+1 \leq s \leq l-1} \mathcal{A}\left(1,2, \ldots, t, s ; w_{1}, w_{2}, \ldots, w_{t}, y_{s}\right) \subseteq \mathcal{D}
$$

$$
|\mathcal{C}| \leq \sum_{0 \leq d \leq l-t-2}\left(\begin{array}{c}
n-d+l-t-2 \\
l-t-2
\end{array}\right)+n^{\frac{1}{2}}\left(\begin{array}{c}
n+l-t-3 \\
l-t-3
\end{array}\right)
$$


and

$$
\begin{aligned}
|\mathcal{A}| & \leq \sum_{0 \leq d \leq l-t-2}\left(\begin{array}{c}
n-d+l-t-2 \\
l-t-2
\end{array}\right)+\left(n^{\frac{1}{2}}+t\right)\left(\begin{array}{c}
n+l-t-3 \\
l-t-3
\end{array}\right) \\
& \leq \sum_{0 \leq d \leq l-t-2}\left(\begin{array}{c}
n-d+l-t-2 \\
l-t-2
\end{array}\right)+2 n^{\frac{1}{2}}\left(\begin{array}{c}
n+l-t-3 \\
l-t-3
\end{array}\right) .
\end{aligned}
$$

Since

$$
\left(\begin{array}{c}
n+l-t-1 \\
l-t-1
\end{array}\right)-\left(\begin{array}{c}
n-1 \\
l-t-1
\end{array}\right)=\sum_{0 \leq d \leq l-t-1}\left(\begin{array}{c}
n-d+l-t-2 \\
l-t-2
\end{array}\right),
$$

we have $|\mathcal{A}|<\left(\begin{array}{c}n+l-t-1 \\ l-t-1\end{array}\right)-\left(\begin{array}{c}n-1 \\ l-t-1\end{array}\right)$ if and only if

$$
2 n^{\frac{1}{2}}\left(\begin{array}{c}
n+l-t-3 \\
l-t-3
\end{array}\right)<\left(\begin{array}{c}
n-1 \\
l-t-2
\end{array}\right) .
$$

By Lemma 3.5,

$$
\begin{aligned}
2 n^{\frac{1}{2}}\left(\begin{array}{c}
n+l-t-3 \\
l-t-3
\end{array}\right) & <\frac{4 n^{l-t-\frac{5}{2}}}{(l-t-3) !} \\
& <\frac{n^{l-t-2}}{(l-t-2) !}\left(1-\frac{l-t-1}{n}\right)^{l-t-2} \\
& <\left(\begin{array}{c}
n-1 \\
l-t-2
\end{array}\right) .
\end{aligned}
$$

Hence, $|\mathcal{A}|<\left(\begin{array}{c}n+l-t-1 \\ l-t-1\end{array}\right)-\left(\begin{array}{c}n-1 \\ l-t-1\end{array}\right)$ and Case 4 is done.

Case 5. Suppose $k=l$. Again, by Theorem 3.1, $\mathcal{A}^{*}\left(1,2, \ldots, t, s ; w_{1}, w_{2}, \ldots\right.$, $\left.w_{t}, y_{s}\right)$ has an independent set of size at least $l-t$ for $s \in[l] \backslash[t]$, if $n \geq$ $(2(l-t-1))^{2^{l-t-2}}+1$. For each $j \in[t]$, let

$$
\begin{gathered}
\mathcal{Q}_{j}=\left\{\mathbf{u} \in \mathcal{A}: \mathbf{u}(i)=w_{i} \text { for all } i \in[t] \backslash\{j\}, \mathbf{u}(j) \neq w_{j},\right. \\
\text { and } \left.\mathbf{u}(i)=y_{i} \text { for all } i \in[l] \backslash[t]\right\} .
\end{gathered}
$$

It follows from Lemma 3.2 that

$$
\mathcal{A}=\mathcal{C} \cup \bigcup_{1 \leq j \leq t} \mathcal{Q}_{j}
$$

Let $n_{j}^{\prime}=n-\sum_{t+1 \leq i \leq l} y_{i}-\left(\sum_{1 \leq i \leq t, i \neq j} w_{i}\right)$. If $n_{j}^{\prime}<0$, then $\left|\mathcal{Q}_{j}\right|=0$. If $n_{j}^{\prime} \geq 0$, then $\left|\mathcal{Q}_{j}\right|=1$. Therefore $|\mathcal{A}| \leq|\mathcal{C}|+t$.

Let

$$
\begin{aligned}
\mathcal{D}=\{\mathbf{u} \in P(n, l): \mathbf{u}(i) & =w_{i} \text { for all } i \in[t] \text { and } \\
\mathbf{u}(i) & \left.=y_{i} \text { for some } t+1 \leq i \leq l\right\} .
\end{aligned}
$$


By equation (2) and Corollary 2.6,

$$
\begin{aligned}
|\mathcal{D}| & \leq \sum_{0 \leq d \leq l-t-1}\left(\begin{array}{c}
n-\sum_{1 \leq i \leq t} w_{i}-d+l-t-2 \\
l-t-2
\end{array}\right) \\
& \leq \sum_{0 \leq d \leq l-t-1}\left(\begin{array}{c}
n-d+l-t-2 \\
l-t-2
\end{array}\right) .
\end{aligned}
$$

Since

we have

$$
\mathcal{C}=\bigcup_{t+1 \leq s \leq l} \mathcal{A}\left(1,2, \ldots, t, s ; w_{1}, w_{2}, \ldots, w_{t}, y_{s}\right) \subseteq \mathcal{D}
$$

and

$$
|\mathcal{C}| \leq \sum_{0 \leq d \leq l-t-1}\left(\begin{array}{c}
n-d+l-t-2 \\
l-t-2
\end{array}\right),
$$

$$
|\mathcal{A}| \leq \sum_{0 \leq d \leq l-t-1}\left(\begin{array}{c}
n-d+l-t-2 \\
l-t-2
\end{array}\right)+t
$$

Since

$$
\left(\begin{array}{c}
n+l-t-1 \\
l-t-1
\end{array}\right)-\left(\begin{array}{c}
n-1 \\
l-t-1
\end{array}\right)=\sum_{0 \leq d \leq l-t-1}\left(\begin{array}{c}
n-d+l-t-2 \\
l-t-2
\end{array}\right)
$$

we have $|\mathcal{A}| \leq\left(\begin{array}{c}n+l-t-1 \\ l-t-1\end{array}\right)-\left(\begin{array}{c}n-1 \\ l-t-1\end{array}\right)+t$. Furthermore, equality holds if and only if $w_{i}=0$ for all $i \in[t]$ and $y_{i}=0$ for all $i \in[l] \backslash[t]$. By the maximality of $\mathcal{A}$,

$$
\mathcal{C}=\bigcup_{s \in[l] \backslash[t]} \mathcal{A}_{s}
$$

and $\mathcal{Q}_{j}=\left\{\mathbf{q}_{j}\right\}$ for all $j \in[t]$. This completes the proof of the theorem.

Acknowledgments. We would like to thank the anonymous referee for the comments that had helped us make several improvements to this paper.

\section{References}

[1] R. Ahlswede and L. H. Khachatrian, The complete intersection theorem for systems of finite sets, European J. Combin. 18 (1997), no. 2, 125-136.

[2] - The diametric theorem in Hamming spaces - optimal anticodes, Adv. in Appl. Math. 20 (1998), no. 4, 429-449.

[3] C. Bey, On cross-intersecting families of sets, Graphs Combin. 21 (2005), no. 2, 161168.

[4] P. Borg, Extremal t-intersecting sub-families of hereditary families, J. London Math. Soc. 79 (2009), no. 1, 167-185.

[5] _ On t-intersecting families of signed sets and permutations, Discrete Math. 309 (2009), no. 10, 3310-3317.

[6] P. Borg and F. C. Holroyd, The Erdös-Ko-Rado property of various graphs containing singletons, Discrete Math. 309 (2009), no. 9, 2877-2885.

[7] F. Brunk and S. Huczynska, Some Erdös-Ko-Rado theorems for injections, European J. Combin. 31 (2010), 839-860. 
[8] P. J. Cameron and C. Y. Ku, Intersecting families of permutations, European J. Combin. 24 (2003), no. 7, 881-890.

[9] A. Chowdhury and B. Patkós, Shadows and intersections in vector spaces, J. Combin. Theory Ser. A 117 (2010), no. 8, 1095-1106.

[10] M. Deza and P. Frankl, On the maximum number of permutations with given maximal or minimal distance, J. Combin. Theory Ser. A 22 (1977), no. 3, 352-360.

[11] D. Ellis, Stability for t-intersecting families of permutations, J. Combin. Theory Ser. A 118 (2011), no. 1, 208-227.

[12] D. Ellis, E. Friedgut, and H. Pilpel, Intersecting families of permutations, J. Amer. Math. Soc. 24 (2011), no. 3, 649-682.

[13] K. Engel and P. Frankl, An Erdös-Ko-Rado theorem for integer sequences of given rank, European J. Combin. 7 (1986), no. 3, 215-220.

[14] P. Erdős, C. Ko, and R. Rado, Intersection theorems for systems of finite sets, Quart. J. Math. Oxford Ser. (2) 12 (1961), 313-320.

[15] P. Frankl, The Erdös-Ko-Rado theorem is true for $n=c k t$, Combinatorics (Proc. Fifth Hungarian Colloq., Keszthely, 1976), Vol. I, pp. 365-375, Colloq. Math. Soc. Jnos Bolyai, 18, North-Holland, Amsterdam-New York, 1978.

[16] P. Frankl and Z. Füredi, Nontrivial intersecting families, J. Combin. Theory Ser. A 41 (1986), no. 1, 150-153.

[17] P. Frankl and N. Tokushige, On r-cross intersecting families of sets, Combin. Probab. Comput. 20 (2011), no. 5, 749-752.

[18] P. Frankl and R. M. Wilson, The Erdös-Ko-Rado theorem for vector spaces, J. Combin. Theory Ser. A 43 (1986), no. 2, 228-236.

[19] C. Godsil and K. Meagher, A new proof of the Erdös-Ko-Rado theorem for intersecting families of permutations, European J. Combin. 30 (2009), no. 2, 404-414.

[20] A. J. W. Hilton and E. C. Milner, Some intersection theorems for systems of finite sets, Quart. J. Math. Oxford (2) 18 (1967), 369-384.

[21] A. J. W. Hilton and C. L. Spencer, A graph-theoretical generalisation of Berges analogue of the Erdös-Ko-Rado theorem, Trends in Graph Theory, Birkhauser Verlag, Basel, Switzerland (2006), 225-242.

[22] F. C. Holroyd, C. Spencer, and J. Talbot, Compression and Erdős-Ko-Rado graphs, Discrete Math. 293 (2005), no. 1-3, 155-164.

[23] F. C. Holroyd and J. Talbot, Graphs with the Erdös-Ko-Rado property, Discrete Math. 293 (2005), no. 1-3, 165-176.

[24] G. Hurlbert and V. Kamat, Erdős-Ko-Rado theorems for chordal graphs and trees, J. Combin. Theory Ser. A 118 (2011), no. 3, 829-841.

[25] P. Keevash, Shadows and intersections: Stability and new proofs, Adv. Math. 218 (2008), no. 5, 1685-1703.

[26] C. Y. Ku and I. Leader, An Erdős-Ko-Rado theorem for partial permutations, Discrete Math. 306 (2006), no. 1, 74-86.

[27] C. Y. Ku and D. Renshaw, Erdös-Ko-Rado theorems for permutations and set partitions, J. Combin. Theory Ser. A 115 (2008), no. 6, 1008-1020.

[28] C. Y. Ku and K. B. Wong, On cross-intersecting families of set partitions, Electron. J. Combin. 19 (2012), no. 4, 9 pp.

[29] _ On r-cross intersecting families of sets, Far East J. Math. Sci. 75 (2013), 295-300.

[30] _ An analogue of the Erdös-Ko-Rado theorem for weak compositions, Discrete Math. 313 (2013), 2463-2468.

[31] _ An analogue of Hilton-Milner theorem for set partitions, J. Combin. Theory Ser. A 120 (2013), 1508-1520.

[32] — On r-cross t-intersecting families for weak compositions, preprint. 
[33] _ An Erdös-Ko-Rado theorem for permutations with fixed number of cycles, Electron. J. Combin. 21 (2014), no. 3, \#P3.16.

[34] C. Y. Ku and T. W. H. Wong, Intersecting families in the alternating group and direct product of symmetric groups, Electron. J. Combin. 14 (2007), no. 3, 9 pp.

[35] B. Larose and C. Malvenuto, Stable sets of maximal size in Kneser-type graphs, European J. Combin. 25 (2004), no. 5, 657-673.

[36] Y.-S. Li and Jun Wang, Erdös-Ko-Rado-type theorems for colored sets, Electron. J. Combin. 14 (2007), no. 1, 9 pp.

[37] M. Matsumoto and N. Tokushige, The exact bound in the Erdös-Ko-Rado theorem for cross-intersecting families, J. Combin. Theory Ser. A 52 (1989), no. 1, 90-97.

[38] A. Moon, An analogue of the Erdös-Ko-Rado theorem for the Hamming schemes $H(n, q)$, J. Combin. Theory Ser. A 32 (1982), no. 3, 386-390.

[39] L. Pyber, A new generalization of the Erdös-Ko-Rado theorem, J. Combin. Theory Ser. A 43 (1986), no. 1, 85-90.

[40] N. Tokushige, A product version of the Erdös-Ko-Rado theorem, J. Combin. Theory Ser. A 118 (2011), no. 5, 1575-1587.

[41] J. Wang and H. Zhang, Cross-intersecting families and primitivity of symmetric systems, J. Combin. Theory Ser. A 118 (2011), no. 2, 455-462.

[42] J. Wang and S. J. Zhang, An Erdös-Ko-Rado-type theorem in Coxeter groups, European J. Combin. 29 (2008), no. 5, 1112-1115.

[43] R. M. Wilson, The exact bound in the Erdös-Ko-Rado theorem, Combinatorica 4 (1984), no. 2-3, 247-257.

[44] R. Woodroofe, Erdős-Ko-Rado theorems for simplicial complexes, J. Combin. Theory Ser. A 118 (2011), no. 4, 1218-1227.

Cheng Yeaw Ku

Department of Mathematics

NATional University of Singapore

117543, Singapore

E-mail address: matkcy@nus.edu.sg

KOK BIN WONG

Institute of Mathematical Sciences

UNIVERSITY OF MALAYA

50603 Kuala Lumpur, Malaysia

E-mail address: kbwong@um.edu.my 